\title{
Correlation of Obesity and Flat-Foot in Haemophilic Adolescents in Karad
}

\author{
Shruti Raphael Dsouzaํㅜ, Gauri Ramesh Gangane², Mandar Ramesh Malawade ${ }^{3}$ \\ 1, 2 Department of Physiotherapy, Krishna Institute of Medical Sciences, Deemed to be University, Malkapur, \\ Karad, Maharashtra, India, ${ }^{3}$ Department of Paediatric Neurosciences, Krishna College of Physiotherapy, \\ Krishna Institute of Medical Sciences, Deemed to be University, Malkapur, Karad, Maharashtra, India.
}

\section{ABSTRACT}

\section{BACKGROUND}

Haemophilic patients are more prone to obesity, due to physical inactivity which is a predisposing factor for structural and functional deformities. In obese people, long term loading has negative impact on lower limb and foot, which causes deformities like flat-foot. In foot, the primary component responsible for absorption and dissipation of forces is medial longitudinal arch and in flat-foot this arch is collapsed. Flat-foot causes pain and discomfort during weight bearing activities. In Haemophilic patients, obesity and flat-foot lead to further postural complications. As no study has been conducted on this topic before, we decided to study on this. The objectives of this study were to assess obesity, flat-foot and co relate obesity and flat-foot in Haemophilic adolescents.

\section{METHODS}

Height and weight of participants were measured to calculate BMI. Total 36 participants were chosen for this study. The subjects were assessed for flat-foot by using Staheli's Plantar Arch Index. Footprints were taken to calculate the plantar index. The Staheli's Plantar Arch Index was measured by dividing the width of central region by width of heel region on footprint, which was calculated as: a line tangent to the medial edge of forefoot and heel region, then a perpendicular line was drawn medially to laterally from mid - point of tangent line in central region of footprint (A) and similarly another perpendicular was drawn from heel region (B). The plantar index was then calculated by dividing value A with value $B$.

\section{RESULTS}

Flat-foot was seen in $25 \%$ population having BMI between 33 - 35. Our data also suggested that age affects the medial arch of foot, the elder population showed increased percentage of flat-foot than the younger group. Individuals with type A haemophilia had increased percentage of flat-foot when compared with type B haemophiliacs, as type A haemophilia is considered severe type.

\section{CONCLUSIONS}

The results show that there is high prevalence of flat-foot in haemophilic patients, this requires rehabilitation and can help prevent further complications.
Corresponding Author: Dr. Gauri Ramesh Gangane, Department of Physiotherapy, Krishna Institute of Medical Sciences, deemed to be University, Malkapur, Karad, Maharashtra, India.

E-mail: gaurigang17@gmail.com

DOI: $10.14260 /$ jemds/2021/411

How to Cite This Article:

Dsouza SR, Gangane GR, Malawade MR. Correlation of obesity and flat foot in haemophilic adolescents in Karad. J Evolution Med Dent Sci 2021;10(27): 2007-2011, DOI:

10.14260/jemds/2021/411

Submission 05-03-2021,

Peer Review 09-05-2021,

Acceptance 15-05-2021,

Published 05-07-2021.

Copyright (C) 2021 Shruti Raphael Dsouza et al. This is an open access article distributed under Creative Commons Attribution License [Attribution 4.0 International (CC BY 4.0)]

\section{KEY WORDS}

Haemophilia, Obesity, Flat-foot 


\section{BACKGROUND}

Haemophilia is a sex-linked bleeding disorder, affecting males and females being carriers. It is characterised by prolonged clotting time. Even a minor trauma can cause excessive bleeding. It is classified into three types based on deficiency of factor involved:

1. Haemophilia A or Classic Haemophilia - occurs due to factor VIII deficiency, affects $85 \%$ of population.

2. Haemophilia B or Christmas disease - occurs due to factor IX deficiency, affects $15 \%$ of population.

3. Haemophilia C - occurs due to deficiency of factor XI, it is very rare disorder. ${ }^{1}$

Due to fear of minor trauma causing excessive bleeding these children avoid physical activity, hence these children are more prone to develop obesity. WHO defines 'Obesity as abnormal or excessive fat accumulation that may impair health'. Obesity is associated with abnormal gait pattern; obesity increases rear foot motion during walking and causes forefoot to abduct significantly more than in normal gait individuals. ${ }^{2}$ As the age progresses and the person gains some weight, the tensile strength of the ligamentous and muscular structures of the foot increases. In children up to $5-6$ yrs. of age the foot development takes place like that of an adult foot type which means all the adipose tissue deposition is lost and they develop an arch on the medial side of foot. However, because of weight gain the excess loading forces on foot lead to collapse of the arch and the foot is pronated, showing symptoms of pain and discomfort in foot which can be problematic with growing age and pain in feet can cause severe discomfort while doing weight bearing activities like walking, standing, running etc. The factors affecting foot development in children can be persistent in adulthood and should be brought to light, so that there can be immediate precautionary measures to avoid these kinds of problems.

A number of factors influence the anatomy and functioning of the feet, one of them being body weight which increases foot discomfort in obese children. ${ }^{3}$ In obese children long term loading effect occurs due to excessive increase in weight bearing forces which negatively affects lower limbs and feet. Feet, as the body's base of support, continually endure often high ground reaction forces generated during activities of daily living. ${ }^{4}$ Foot plays an important role in biomechanical alignment of body and hence alteration in foot biomechanics leads to deformities like flat-foot. ${ }^{3}$ Flat-foot also called as Pes Planus is defined as 'collapse in the medial and longitudinal arches of foot'. In flat-foot sole of foot comes into complete or near - complete contact with ground during weight bearing activities which causes pain and discomfort in foot. Flat-foot is a complex disorder, having various symptoms and varying degrees of deformity and disability. The medial longitudinal arch is a vital factor that influences shape and function of foot. Haemophilic patients are prone to develop obesity, which causes negative effect on their musculoskeletal system and causes other co-morbidities along with flat-foot, which further adds to their problems. Haemophilic patients are more prone to obesity, due to physical inactivity which is a predisposing factor for structural and functional deformities. In obese people, long term loading has negative impact on lower limb and feet, which causes deformities like flat-foot. In foot, the primary component responsible for absorption and dissipation of forces is medial longitudinal arch and in flatfoot this arch is collapsed. Flat-foot causes pain and discomfort during weight bearing activities. In Haemophilic patients, obesity and flat-foot leads to further postural complications. As no study has been conducted on this topic before, this made us study this topic. The objectives of this study were to assess obesity, flat-foot and co relate obesity and flat-foot in Haemophilic adolescents.

\section{METHODS}

This study was analytical, it was done to co relate obesity and flat-foot in Haemophilic adolescents. This study was carried out in Krishna College of Physiotherapy, Karad, for a duration of 6 months from December 2019 to May 2020. Total 36 participants participated in this study, Sample size was calculated using the formula $\mathrm{n}=4 p q \div l^{2}$.

The participants included in this study were Haemophilic adolescents aged between 13 and 19 years and having BMI above $30 \mathrm{~kg} / \mathrm{m}^{2}$. Individuals with lower limb amputation, psychologically disturbed and mentally challenged were excluded. The participants were assessed for obesity by calculating BMI using Standard Quetelet Index protocol - BMI $=$ Weight $/$ Height $\left(\mathrm{kg} / \mathrm{m}^{2}\right)$. Height and weight of participants were measured in light clothes and without shoes using standard apparatus.

A calibrated weighing scale was used to measure weight. Height was measured by using a measuring tape which was fixed to the wall. Height was measured while the participant stood with heels, buttocks, shoulder, and occiput touching the vertebral tape. For participants of age group 13 - 19 years, the CDC's clinical growth chart was used to determine reference percentile for BMI specific to age and gender. The subject was assessed for flat-foot by using Staheli's Plantar Arch Index. Footprints were taken to calculate the plantar index. ${ }^{5}$ The participants were asked to wash their feet and which was later dried properly. Their foot was impregnated with the stamp ink and the impression was taken using the white paper with bare foot relaxed in anatomical position. The principle of the Staheli's Plantar Arch Index is that the height of the arch is related to footprint. The Staheli's Plantar Arch Index was measured by dividing the width of central region by width of heel region on footprint, which was calculated as: a line tangent to the medial edge of forefoot and heel region, then a perpendicular line was drawn medially to laterally from mid - point of tangent line in central region of footprint (A) and similarly another perpendicular was drawn from heel region (B). The plantar index was then calculated by dividing value $A$ with value $B$.

\section{Statistical Analysis}

It was done by using software SPSS version by using university code. Arithmetic mean and standard deviation was calculated for each outcome measure. MS Excel was used for drawing various graphs with given frequencies and the various percentages that were calculated with the software. Paired $t$ - test was used and the $p$ value was less than 0.0001 
which meant that our co relation was not significant statistically.

\section{RESULTS}
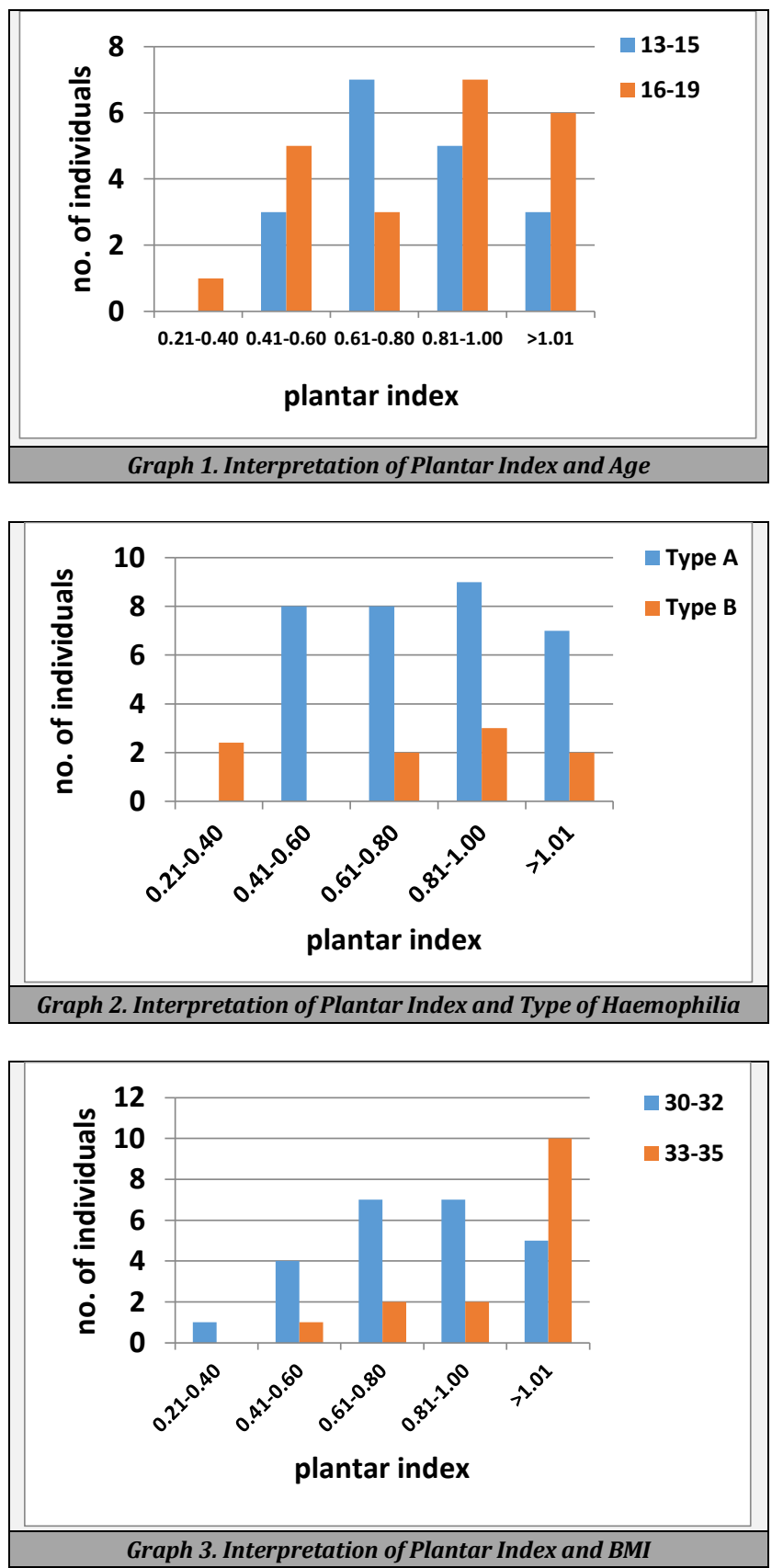

According to statistical analysis (Graph 1) patients in the age group of 16 - 19 years having plantar index above 1 were 15 $\%$ and patients in the age group of $13-15$ years having plantar index above 1 were $7.5 \%$. Patients in the age group of 16 - 19 years showed considerable increase in plantar index with a difference of $7.5 \%$.

According to statistical analysis (Graph 2), patients in the age group of 16 - 19 years having plantar index above 1 were $15 \%$ and patients in the age group of $13-15$ years having plantar index above 1 were $7.5 \%$. Patients in the age group of 16 - 19 years showed considerable increase in plantar index with a difference of $7.5 \%$.
According to statistical analysis (Graph 3), patients in the age group of 16 - 19 years having plantar index above 1 were $15 \%$ and patients in the age group of $13-15$ years having plantar index above 1 were $7.5 \%$. Patients in the age group of 16 - 19 years showed considerable increase in plantar index with a difference of $7.5 \%$.

\section{DISCUSSION}

This study was conducted to find out co-relation of obesity and flat foot in haemophilic adolescents. Participants having BMI above 33 showed considerable increase in plantar index when compared to participants having BMI between $30-32$, this shows that there exists an association between BMI and plantar index. When plantar index was compared to type of Haemophilia it showed that participants with type A Haemophilia had a higher value of plantar index than participants with type B Haemophilia. Participants were divided into two groups according to age: $13-15$ years \& 16 19 years, the elderly group (16 - 19) showed considerable increase in plantar index, this shows that age has an effect on plantar index. Among all the three categories, BMI is the one which can be managed by physical activity and lifestyle modification which helps in reducing complications of musculoskeletal system like flat-foot, osteoarthritis, joint damage due to intra-articular bleeding and long term complications of cardiovascular system. The aetiology of obesity in haemophilic and non - haemophilic population remains same. In haemophilic patients, obesity may lead to cardiovascular events and the risk increases as age progresses. The result of cardiovascular disease may be aggravated by bleeding tendency in haemophilic population hence, it is important to prevent it. This study shows that greater the BMI higher is the risk for flat-foot, which affects the overall posture and gait of an individual.

In a study conducted in Mississippi, USA, the aim of this study was to find out prevalence of obesity and overweight in haemophilic adults and children. The prevalence of obesity in the adult haemophilic patients was $36 \%$ and additional $32 \%$ were overweight. The results show that there is a high prevalence of overweight and obesity in haemophilic population. ${ }^{6}$ In a study conducted in Chennai, Tamil Nadu, the aim of this study was to determine effects of obesity on foot structure. The overall prevalence of flat-foot in obese individuals was $44 \%$. As per our results there has been a strong co-relation between overweight and obesity or obese and flat-foot presence, which indicates that there exists an association. ${ }^{7}$

The studies identified through Paediatric review showed increase in musculoskeletal problems in overweight and obese children which was expressed as pain due to change in knee and ankle joint. These changes have further shown negative impact on motor performance, muscle strength, balance and walking because of change in plantar flexion during swing phase of walking, resulting in flatter foot. Previous studies suggested that these structural changes in foot anatomy may have affected the functional capacity of the medial longitudinal arch. The existing reports suggest that, the weight of the individuals is directly proportional to the lower plantar arch height and that there exists a strong 
relationship between weight and flat foot grade, that is, as the BMI increases, the grade level of flat foot also increases. The lower arch height in obese individuals shows that flat - feet would be a characteristic feature in obese individuals which causes change in foot anatomy. ${ }^{8}$

Studies have been conducted where it is proven that there is marked difference in foot loading in overweight children than normal weight children. There is significantly greater peak pressure, peak forces and increased force time and pressure time under mid-foot and $2^{\text {nd }}-5^{\text {th }}$ metatarsals of overweight and obese children. These reports show that overweight and obese children can develop foot discomfort in future due to increased-pressure and forces acting on the developing musculoskeletal structure of foot. As there is longer duration of foot loading pattern found in obese and overweight individuals, there is increased ground contact of mid-foot during walking, which is due to excessive adipose tissue around medial longitudinal arch which results in increased loading, and this excess load can cause the foot to pronate. This suggests that increased loading can have negative effect on biomechanical function of foot in overweight and obese children. Significant change was found even when peak forces were normalized to body mass. ${ }^{9}$

In a study conducted in Amsterdam, Netherland, the Haemophilic overweight individuals who were prone to arthritic and chronic joint problems when compared to normal weight individuals showed that overweight and obesity further aggravated joint bleeding and compromised joint function in Haemophilic patients as they frequently suffered from arthropathy. Regardless of severity of Haemophilia a strong association between BMI and limitation in joint range of motion is found. Obesity is directly related to a number of other musculoskeletal and systemic conditions and is responsible for a lot of disabilities and affects quality of life. ${ }^{10}$

A study conducted on joint range of motion limitation among Haemophiliacs based on frequency of bleeds suggested that chronic bleeding into the joints produces an inflammatory response that leads to arthropathy of joints. They observed that there was significant limitation in joint range of motion (ROM) associated with number of bleeds. The limitation in joint range of motion was seen in individuals with severe haemophilia when compared to mild and moderate haemophilic patients, the possible explanation for this could be high frequency of bleeding in severe haemophilic patients when compared to others.

It was also observed that increase in age and BMI were also associated with range of motion limitation in individuals with moderate haemophilia. ${ }^{11}$ In a previous study conducted in Canada, they assessed whether haemophilia negatively affects balance control in male adolescents, the objective of this study was whether the haemophilic adolescents had increased COP speed, which could be interpreted as decreased postural stability, even when vision and proprioception were altered. The results showed that haemophilic adolescents had more postural instability when compared to normal - healthy individuals. Balance control is an essential part in daily life, which is important for the movements we perform. ${ }^{12}$ People with Haemophilia should be given activities in a controlled manner so that they remain physically active and have no fear about having bleeds in joints or anywhere in body. 4
Several developmental factors including BMI have an effect on child's overall health and lower limb alignment; the other factors cannot be modified but increase in BMI is manageable and can prevent the child from other severe health issues. Childhood obesity continues into adult life and may have a negative-impact on the individual's quality of life, increasing chances of having comorbidities like myocardial infarction, stroke, hypertension, diabetics, etc. Foot pain and discomfort is usually associated with increased tissue stress which may cause complications in particular weight bearing physical activities. So the consequences of overweight and obesity on lower limb and foot should be managed properly and a multidisciplinary care must be ensured. Some individuals with flat-foot may never have problem while engaging in sports activities like running or any other. On the other hand some individuals may have arch pain, swelling around the ankle or sometimes have difficulty in standing in same position which are considered as signs and symptoms of flat-foot.

\section{CONCLUSIONS}

The results show that there is high prevalence of flat-foot in haemophilic patients, which affects gait pattern, and increase their risk to develop further complications in lower limb. In haemophilic individuals due to high frequency of bleeds it negatively affects range of motion. Among the three categories, BMI and type of haemophilia had a strong association with plantar index, this indicates that plantar arch height is directly proportional to BMI, the higher the BMI the lower is the plantar arch height.

\section{Limitations and Suggestions}

This study was conducted on limited sample size and hence the results may vary for large sample size. Also the data collected was from a small geographical area. Study was done only on participants aged between 13 and 19 years and did not cover all the age groups.

Data sharing statement provided by the authors is available with the full text of this article at jemds.com.

Financial or other competing interests: None.

Disclosure forms provided by the authors are available with the full text of this article at jemds.com.

\section{REFERENCES}

[1] Sembulingam K, Sembulingam P. Essentials of medical physiology. $6^{\text {th }}$ edn. Jaypee Brothers Medical Publishers Ltd 2012.

[2] Walsh M, Macgregor D, Stuckless S, et al. Health-related quality of life in a cohort of adult patients with mild Haemophilia A. J Thromb Haemost 2008;6(5):755-61.

[3] Zonfrillo MR, Seiden JA, House EM, et al. The association of overweight and ankle injuries in children. Ambul Pediatr 2008;8(1):66-9.

[4] Thabi HA, Beausoleil S, Hue 0. The effects of haemophilia on the postural control of adolescents. J Funct Morphol Kinesiol 2017;2(3):24. 
[5] Hernandez AJ, Kimura LK, Laraya MH, et al. Calculation of staheli's plantar arch index and prevalence of flat feet: a study with 100 children aged 5-9 years. Acta Ortop Bras 2007;15(2):68-71.

[6] Majumdar S, Morris A, Gordon C, et al. Alarmingly high prevalence of obesity in haemophilia in the state of Mississippi. Haemophilia 2010;16(3):455-9.

[7] Shree S, Revathi S, Thiyagarajan A, et al. Does obesity cause flat foot. J Obes Ther 2018;2(1):1000106.

[8] Smith SM, Sumar B, Dixon KA. Musculoskeletal pain in overweight and obese children. Int J Obes (Lond) 2014;38(1):11-5.
[9] Cousins SD, Morrison SC, Drechsler WI. Foot loading patterns in normal weight, overweight and obese children aged 7 to 11 years. J Foot Ankle Res 2013;6(1):1-6.

[10] Hofstede FG, Fijnvandraat K, Plug I, et al. Obesity: a new disaster for haemophilic patients? A nationwide survey. Haemophilia 2008;14(5):1035-8.

[11] Dowling AM, Steele JR, Baur LA. What are the effects of obesity in children on plantar pressure distributions? Int J Obes Relat Metab Disord 2004;28(11):1514-9.

[12] Ganu SS, Panhale V. Effect of obesity on arch index in young adults. Online J Health Allied Sci 2012;11(4):1-3. 